\title{
EFICIÊNCIA DE ADUBAÇÃO NITROGENADA CAJUZINHO-DO-CERRADO Anacardium othonianum RIZZINI
}

Valéria Lima da Silva ${ }^{1}$, Alessandra Conceição de Oliveira ${ }^{2}$, Weslian Vilanova da Silva ${ }^{3}$, Sônia França $\operatorname{Costa}^{3}$, Carlos Cesar Silva Jardim ${ }^{3}$

\footnotetext{
${ }^{1}$ Pós-graduando (Especialização) Agroecologia, Instituto Federal de Mato Grosso / IFMT, Barra do Garças MT. Email: valeria.silva21@ hotmail.com

${ }^{2}$ Docente do Departamento de Agronomia, Irrigação e Drenagem - Universidade do Estado de Mato Grosso / UNEMAT, Nova Xavantina - MT. ${ }^{3}$ Graduandos (as) em Engenharia Agronômica- Universidade do Estado de Mato Grosso/ UNEMAT, Nova Xavantina- MT.
}

RESUMO: No presente trabalho teve como objetivo avaliar o desenvolvimento de mudas cajuzinho-do-cerrado, sob diferentes doses de nitrogênio, em um viveiro telado com $50 \%$ de luminosidade. O experimento foi conduzido no viveiro da Universidade do Estado de Mato Grosso (UNEMAT), de Nova Xavantina-MT. A produção de mudas foi realizada em sacos de polietileno com as dimensões $14 \times 20 \mathrm{~cm}$, com capacidade para um litro de substrato de 2:1 (Solo/Esterco), sendo que o esterco bovino foi curtido, peneirado e misturado. Adotouse o delineamento de blocos casualizados com 4 blocos x 5 tratamentos $(0,150 ; 300 ; 450$ e $600 \mathrm{mg} \cdot \mathrm{dm}^{-3}$ ), cada parcela contendo 10 plantas e foram analisados apenas as oito plantas centrais em cada tratamento. Diferentes doses de Nitrogênio parceladas em três aplicações, sendo as mesmas diluídas em água e aplicadas posteriormente. O intervalo entre aplicações era a cada 15 dias. A solução foi aplicada com auxílio de uma seringa com $20 \mathrm{ml}$ por saquinho. Foi realizada uma adubação de cobertura a cada 15 dias, sendo utilizados: supersimples $(198 \mathrm{mg})$, cloreto de potássio $(23 \mathrm{mg})$, zinco $(0,21 \mathrm{mg})$ e boro $(0,5 \mathrm{mg})$. As variáveis analisadas foram: altura da parte aérea, comprimento da raiz, número de folhas, diâmetro do caule, peso da massa fresca das folhas, caule e raiz e massa seca das folhas, caule e raiz. A avaliação estatística do experimento foi realizada pelo programa computacional SISVAR 5.1 - Sistema para Análise de Variância, e foram comparadas ao teste de regressão $1 \%$ e $5 \%$ de probabilidade.

Palavras-chave: Análise crescimento. Fertirrigação. Ureia. Caju.

\section{NITROGEN FERTILIZER EFFICIENCY IN CLOSED BOX}

ABSTRACT: In the present work, the objective of this study was to evaluate the development of cassava seedlings of the cerrado, under different nitrogen rates, in a screened nursery with $50 \%$ luminosity. The experiment was conducted in the nursery of the State University of Mato Grosso (UNEMAT), Nova Xavantina-MT. The production of seedlings was carried out in polyethylene bags with dimensions $14 \times 20 \mathrm{~cm}$, with capacity for 1 liter of substrate of 2: 1 (Soil / Spraying), being the bovine manure was tanned, sieved and mixed. A randomized block design with 4 blocks x 5 treatments $(0.150,300,450$ and 600

Cultura Agronômica, Ilha Solteira, v.26, n.1, p.95-102, 2017 
mg. $\mathrm{dm}^{-3}$ ) was used, each plot containing 10 plants and only eight plants were analyzed in each treatment. Different doses of Nitrogen were divided in three applications, the same being diluted in water and applied later. The interval between applications was 15 days. The solution was applied with a $20 \mathrm{ml}$ syringe per bag. Coverage was applied every 15 days, using super simples $(198 \mathrm{mg})$, potassium chloride $(23 \mathrm{mg})$, zinc $(0.21 \mathrm{mg})$ and boron $(0.5$ $\mathrm{mg}$ ). The analyzed variables were: shoot height and root length, number of leaves, stem diameter, weight of fresh leaf mass, stem and root and dry mass of leaves, stem and root. The statistical evaluation of the experiment was performed by the SISVAR 5.1 - System for Analysis of Variance, and were compared to the $1 \%$ and $5 \%$ probability regression test.

Key words: Growth analysis. Fertigation. Urea. Cashew.

\section{INTRODUÇÃO}

O cajuzinho -do- cerrado (Anacardium othonianum Rizzini) está entre as espécies que apresentam grande potencial econômico para utilização em sistemas de produção agrícola, destaca-se o caju arbóreo do Cerrado, conhecido popularmente por cajuzinho do cerrado sendo bastante útil às populações. É muito difundido na medicina popular e o seu pseudofruto pode ser consumido in natura ou na forma de sucos, doces, geleias, sorvetes e compotas, sua amêndoa torrada é comestível e bastante saboreada (ALMEIDA et al.,1998).

No entanto, apesar das diversas utilidades das frutíferas do Cerrado a situação dessas espécies se agravam ao passo da grande pressão que o homem vem causando sobre esse Bioma na busca da expansão agrícola mecanizada, sem levar em consideração o estudo e o conhecimento que priorizem o manejo sustentável da biodiversidade presente neste ecossistema (BELO, 2014). As práticas culturais para as espécies de cajueiros nativos na região Centro-Oeste ainda não foram determinadas e a exploração dessa espécie ainda é limitada tanto em estudos sobre a cultura como a adubação.

A produção de mudas é considerada uma etapa importante no sistema produtivo, pois a qualidade da muda influência diretamente no desempenho final das plantas nos campos de produção. Para a produção de mudas em viveiros comerciais é de suma importância que o viveirista adquira sementes de instituições que comercializam sementes certificadas, podendo também obter sementes do próprio pomar, se o mesmo apresentar boas condições fitossanitárias (CARVALHO et al., 2015). A escolha de uma cultivar adaptada à região de cultivo e que apresente boas características produtivas é fundamental para o sucesso da atividade.

Além da escolha adequada da cultivar, a utilização de substratos de qualidade é fundamental para garantir o crescimento satisfatório das mudas. Os substratos exercem a função de sustentação das plantas, proporcionam o crescimento das raízes e fornecem as quantidades adequadas de ar, água e nutrientes às plantas (SILVA et al., 2001). A qualidade 
do substrato depende da sua formulação, podendo, inclusive, reduzir adubações complementares, diminuindo o custo de produção da muda (RODRIGUES, 2012).

O nitrogênio é o nutriente essencial mais exigido pelas plantas e responsável por diversas reações biológicas, sendo o seu manejo um dos mais complexos dentre os macronutrientes (MARSCHNER, 1995; FAGAN et al., 2007). O estado nutricional é componente primário para o controle de doenças, e desta forma, o equilíbrio nutricional do vegetal é considerado um dos principais fatores responsáveis por mecanismos de defesa frente aos fatores bióticos (GOMES et al., 2007). O desequilíbrio nutricional relacionado com o nitrogênio pode resultar em aumento da severidade de determinadas doenças (TOMAZELA, 2006; LIMA et al., 2010). Porém, devido à multiplicidade de reações química e biológica, à dependência das condições ambientais e ao seu efeito no rendimento das culturas, o nitrogênio apresenta maiores dificuldades de manejo na produção agrícola mesmo em propriedades tecnicamente orientadas. As práticas culturais para as espécies de cajueiros nativos na região Centro-Oeste ainda não foram determinadas e a exploração dessa espécie ainda é limitada tanto estudos sobre a cultura como a adubação nitrogenada.

Diante do exposto, objetivou-se, com este trabalho, avaliar o desenvolvimento de mudas de cajuzinho-do-cerrado com diferentes doses de nitrogênio.

\section{MATERIAL E MÉTODOS}

O trabalho foi realizado na área experimental da Unemat - Universidade do Estado do Mato Grosso, no período de novembro de 2015 a janeiro de 2016. O experimento foi instalado e conduzido em telado com $50 \%$ de sombreamento. Para a instalação do experimento retirou-se as sementes, destacando-as de pseudofrutos maduros de cajuzinhodo-cerrado, em meados de outubro e novembro em uma propriedade particular, em Nova Xavantina/MT.

Foi realizada uma seleção das sementes, separando aquelas fora do padrão (atacadas por pragas, deformadas, etc.). Em seguida fez-se um teste de densidade com as sementes, colocando-as em recipiente com água, por 24 horas antes do plantio para se obter uma germinação padrão das sementes. Posteriormente, foram secas sobre papel toalha naturalmente. As sementes foram armazenadas em sacos de papel por duas semanas numa temperatura ambiente.

A produção de mudas foi realizada em sacos de polietileno com as dimensões $14 \times 20$ $\mathrm{cm}$, com capacidade para um litro de substrato de 2:1 (Solo/Esterco), sendo que o esterco bovino foi curtido, peneirado e misturado. Cerca de 25 dias após a emergência, as plantas foram desbastadas deixando apenas as mais vigorosas por recipiente.

Adotou-se o delineamento de blocos casualizados com 4 blocos x 5 tratamentos $\left(0,150 ; 300 ; 450\right.$ e $\left.600 \mathrm{mg} \cdot \mathrm{dm}^{-3}\right)$, cada parcela contendo 10 plantas e foram analisados apenas as oito plantas centrais em cada tratamento.

Cultura Agronômica, Ilha Solteira, v.26, n.1, p.95-102, 2017 
Foi realizado análise de solos apresentados na (Tabela 1) abaixo:

Tabela 1. Resultados da análise química e física do substrato utilizado no experimento de mudas de Cajueiro. Nova Xavantina, MT.

\begin{tabular}{|c|c|c|c|c|c|c|c|c|c|c|c|}
\hline \multirow[t]{2}{*}{$\begin{array}{c}\mathrm{pH} \\
\mathrm{CaCl}\end{array}$} & $\mathrm{Ca}$ & $\mathrm{Mg}$ & $\mathrm{Al}$ & $\mathrm{H}+\mathrm{Al}$ & SB & $\mathrm{K}$ & $\mathrm{P}$ & CTC & V & M.O. Argila Silte & Areia \\
\hline & ----- & ---- & $\mathrm{cmol}_{\mathrm{c}}$ & $\mathrm{dm}^{-3}$ & ----- & $\mathrm{mg}$ & $\mathrm{lm}^{-3}$ & $\mathrm{cmol}_{\mathrm{c}} \mathrm{dm}^{-3}$ & $\%$ & 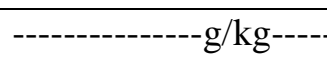 & ------ \\
\hline 7,8 & 3,21 & 2,0 & 0,12 & 2,20 & 12,6 & 7,35 & 13,9 & 12,6 & 85,1 & $\begin{array}{lll}33,70 & 192,0 & 50\end{array}$ & 758,0 \\
\hline
\end{tabular}

Foi realizada a adubação nitrogenada parceladas em três aplicações, sendo as mesmas diluídas em água e aplicadas posteriormente. O intervalo entre aplicações era de 15 dias. A solução foi aplicada com auxílio de uma seringa com $20 \mathrm{ml}$ por saquinho.

Foi realizada uma adubação de cobertura a cada 15 dias, sendo utilizados: supersimples $(198 \mathrm{mg})$, cloreto de potássio $(23 \mathrm{mg})$, zinco $(0,21 \mathrm{mg})$ e boro $(0,5 \mathrm{mg})$. Após serem pesados foram diluídos em água e aplicados com um regador com capacidade de $8 \mathrm{~L}$. As análises de produtividade seguiram os seguintes parâmetros: para a altura linear das plantas e comprimento do sistema radicular, mediu-se do colo até o ápice da parte aérea e do colo ao extremo da raiz, respectivamente, obtendo a média por planta em centímetro com o auxílio de régua graduada. $\mathrm{O}$ diâmetro do colo foi obtido com auxílio de um paquímetro digital obtendo-se a média por planta em milímetros. $\mathrm{O}$ número de folhas foi determinado a partir da expansão das folhas de $1 \mathrm{~cm}$ (número/planta). A determinação da matéria seca foi realizada da seguinte forma: Separou-se a raiz da parte aérea, com auxílio de tesoura de poda; lavou-se em água corrente, colocando o material em sacos de papel e etiquetados; colocou-se o material para secar em estufa com circulação forçada de ar a uma temperatura de $65^{\circ} \mathrm{C}$ por 72 horas, até atingirem massas constantes. Procedeu-se à pesagem em balança analítica $(0,01 \mathrm{~g})$ e o resultado foi expresso em gramas por planta. Obteve-se a massa seca total com a soma das médias da raiz e parte aérea.

A avaliação estatística do experimento foi realizada pelo programa computacional SISVAR 5.1 - Sistema para Análise de Variância, e foram comparadas ao teste de regressão $1 \%$ e $5 \%$ de probabilidade.

\section{RESULTADOS E DISCUSSÃO}

Em outros trabalhos semelhantes a aplicação de $\mathrm{N}$, utilizando-se uréia promoveu efeitos significativos em todas as características de crescimento avaliadas. Quando houve acréscimos nas doses de $\mathrm{N}$ aplicados ocorreu um aumento da altura dos porta-enxertos. Os porta-enxertos de cajueiro obteve altura máxima estimada de 25,74 cm (MENDONÇA et al., 2010). 


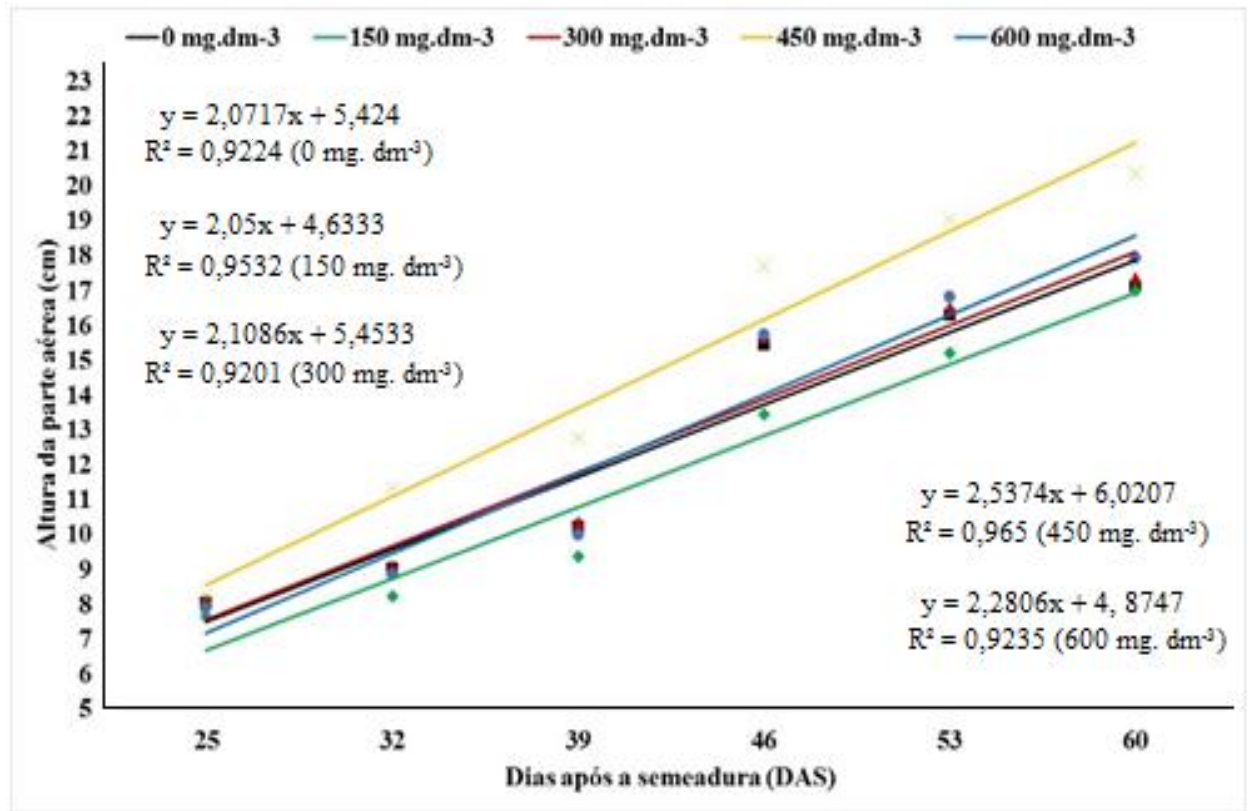

Figura 1. Altura de cajuzinho-do-cerrado em função das doses de nitrogênio. Nova Xavantina - MT, 2016.

Na Figura 1, verifica-se que a dose de $450 \mathrm{mg} \cdot \mathrm{dm}^{-3}$ proporcionou maior altura da parte aérea, $21 \mathrm{~cm}$ em relação as outras dosagens. As dosagens de 150, 300 e 600 mg.dm ${ }^{-3}$ não obtiveram respostas significativas. Teixeira et al. (2004), verificaram que a utilização de sulfato de amônio em cobertura proporcionou, em média, um incremento de $112,5 \%$ na altura das mudas de mamoeiro quando comparadas com mudas que não foram adubadas com $\mathrm{N}$ em cobertura independente do substrato utilizado.

A dose elevada de $600 \mathrm{mg} \cdot \mathrm{dm}^{-3}$ de $\mathrm{N}$ propiciou uma redução da altura da parte aérea. No entanto, foi observado comportamento quadrático para esta variável, em função do aumento de doses de nitrogênio, em mudas de mamoeiro Formosa (MENDONÇA et al., 2009).

Analisando as respostas das doses de $\mathrm{N}$ sobre o diâmetro do caule (Figura 2), verificou-se que o maior valor desta característica foi de $5,1 \mathrm{~mm}$ na dose estimada de 450 mg.dm ${ }^{-3}$ de N no substrato. 


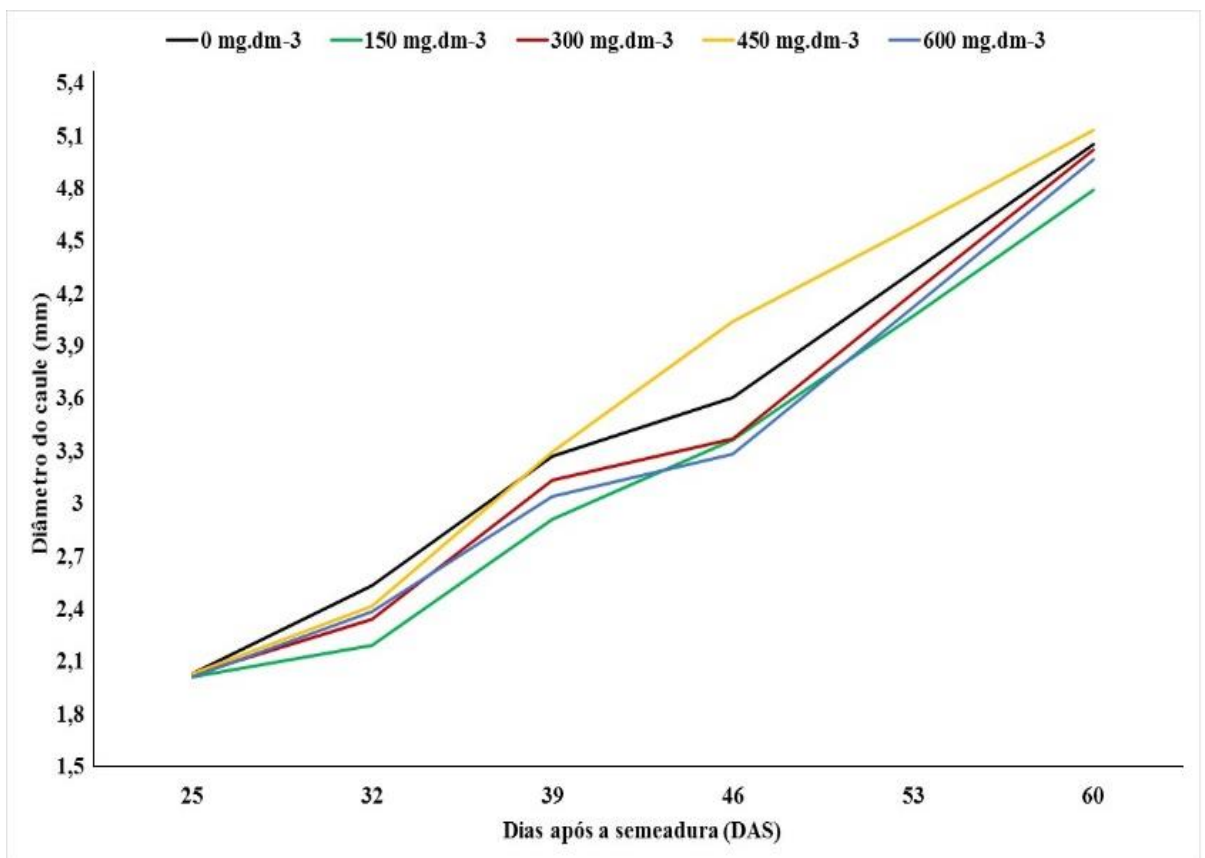

Figura 2. Diâmetro do caule de cajuzinho-do-cerrado em função das doses de nitrogênio. Nova Xavantina - MT, 2016.

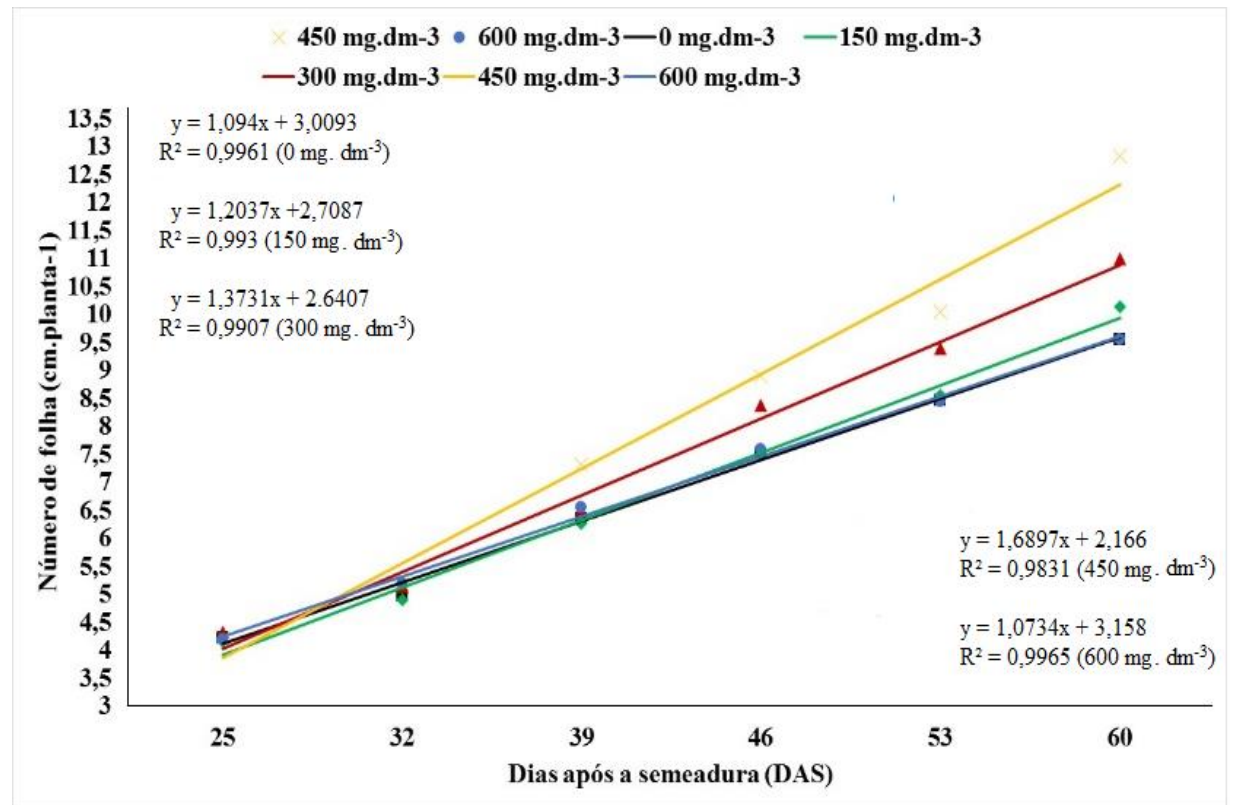

Figura 3. Número de folhas do caule de cajuzinho-do-cerrado em função das doses de nitrogênio. Nova Xavantina - MT, 2016.

Na Figura 3, observa-se que o número de folhas teve um incremento até a dosagem de $450 \mathrm{mg} \cdot \mathrm{dm}^{-3} \mathrm{~N}$, onde foi observado um valor de 12,5 unidades de folhas por muda de caju do cerrado. O nitrogênio é um dos principais agentes de crescimento das plantas e de desenvolvimento foliar. Dosagens elevadas, $600 \mathrm{mg} \cdot \mathrm{dm}^{-3}$ de $\mathrm{N}$ promoveram efeitos depressivos nas mudas, propiciando uma redução do número de folhas por plantas. Tal resultado também foi observado em mudas de mamoeiro Formosa com o aumento da 
adubação nitrogenada em cobertura (MENDONÇA et al., 2009). Com o aumento das dosagens de nitrogênio em cobertura no substrato, ocorreu uma redução linear do comprimento da parte aérea. Esse decréscimo pode estar associado a algum desequilíbrio nutricional causado pelo excesso do $\mathrm{N}$ nas plantas. Este efeito, segundo DECARLOS NETO et al. (2002), pode ser decorrente da redução do pH do substrato, por meio da liberação de $\mathrm{H}^{+}$produzidos durante o processo de nitrificação da uréia aplicada. Apesar da importância real e potencial que o cajueiro apresenta, principalmente na região Nordeste, verifica-se a existência de poucas informações científicas a respeito desta frutífera, principalmente, sobre adubação na formação da muda. Já em outras espécies a adubação na formação da muda já é bem estudada. Segundo Dias et al. (2012), trabalhando com mudas de goiabeira, observou em que doses maiores foram positivas, obtendo um aumento tanto de altura de planta, diâmetro do caule, número de folhas, índice SPAD (Soil Plant Analysis Development), massa seca de folhas, caule, total, IQD (índice de qualidade de Dickson) e acúmulo de N.

Portanto Mendonça et al. (2004), concluíram que a utilização de adubações nitrogenada em cobertura em dose de até $2.000 \mathrm{mg} \mathrm{dm}^{-3} \mathrm{~N}$ no substrato garante melhor qualidade na formação de mudas de maracujazeiro amarelo e doses elevadas promoveram efeitos depressivos nas mudas.

\section{CONCLUSÃO}

Conclui-se que a adubação nitrogenada em Anacardium othonianum Rizzini torna-se necessária para o maior crescimento e desenvolvimento das mudas. A utilização de adubação em dose de até $450 \mathrm{mg}^{-\mathrm{dm}^{-3}}$ de $\mathrm{N}$ no substrato pode ser recomendada para a produção de mudas de cajuzinho-do-cerrado.

\section{REFERÊNCIAS BIBLIOGRÁFICAS}

ALMEIDA, S. P.; PROENÇA, C. E. B.; SANO, S. M.; RIBEIRO, J, F. Cerrado: espécies vegetais úteis. Planaltina, EMBRAPA-CPAC, 1998. 464 p.

BELO, A. P. M. Precocidade de produção, caracterização fenológica, biométrica e ocorrência de antracnose no caju arbóreo do Cerrado (Anacardium othonianum Rizz.) 2014. 50f. Dissertação (Mestrado em Agronomia) - Universidade Federal de Goiás, Goiânia, 2014.

DECARLOS NETO, A.; SIQUEIRA, D. L.; PEREIRA, P. R. G.; ALVAREZ V, V. H. Crescimento de porta enxertos de citros em tubetes influenciados por doses de N. Revista Brasileira de Fruticultura, Jaboticabal, v. 24, n. 2, p.199-203. 2002.

DIAS, M. J. T.; SOUZA, H. A; NATALE, W.; MODESTO, V. C; ROZANE, D. E. Adubação com nitrogênio e potássio em mudas de goiabeira em viveiro comercial. Semina: Ciências Agrárias, Londrina, v. 33, supl. 1, p.2837-2848, 2012. 
GOMES, R. F.; SILVA, A. G.; ASSIS, R. L.; PIRES, F. R. Efeito de doses e da época de aplicação de nitrogênio nos caracteres agronômicos da cultura do milho sob plantio direto. Revista Brasileira de Ciências do Solo, Viçosa, v. 31, n. 5, p.931-938, 2007.

LIMA, L. M.; TORRES, POZZA, E. A.; TORRES, H. N.; POZZA, A. A. A.; SALGADO, M.; PFENNING, L. H. Relação nitrogênio/potássio com mancha de Phoma e nutrição de mudas de cafeeiro em solução nutritiva. Tropical Plant Pathology, Lavras, v. 35, n. 4, p.223-228, 2010.

MARSCHNER, H. Mineral nutrition of higher plants. 2. ed. London. Academic Press, 1995,889 p.

MENDONCA, V.; ARRUDA, N. A. A.; TEIXEIRA, G. A.; SOUZA, H. A.; GURGEL, R. L. S.; FERREIRA, E. A.; RAMOS, J. D. Adubação nitrogenada e diferentes substratos no desenvolvimento de mudas de maracujazeiro amarelo. In: CONGRESSO DA PÓS GRADUAÇÃO DA UFLA, 13., 2004, Lavras. Anais... Lavras: UFLA, 2004, UFLA. CD ROM.

MENDONÇA, V.; COSTA, M. S.; MENDONÇA, L. F. M.; BISCARO, G. A.; FREITAS, P. S. C.; PEREIRA, E. C.; LEITE, G. A. Doses crescentes de nitrogênio sobre o crescimento inicial de porta-enxertos de cajueiro gigante. Revista Agrarian, Dourados, v. 3, n. 8, p.95-103, 2010.

MENDONÇA, V.; RAMOS, J. D.; ABREU, N. A. A.; TEIXEIRA, G. A.; SOUZA, H. A. GURGEL, R. L. S.; ORBES, M. Y. Adubação nitrogenada em cobertura e substratos na produção de mudas de mamoeiro 'formosa'. Ciência Agrotecnologia, Lavras, v. 33, n. 3, p.668-675, 2009.

TEIXEIRA, J. D.; PEIXOTO, J. R.; VASCONCELOS, D. R.; PIRES, M. C.; FLEURY, R. C.; MELO, B. Desenvolvimento de mudas de mamoeiro em diferentes substratos químicos e orgânicos, sob telado. In: CONGRESSO BRASILEIRO DE FRUTICULTURA, 18., 2004, Florianópolis. Anais... Florianópolis: SBF, 2004. CD-ROM.

TOMAZELA, A. L, FAVARIN, J. L; FANCELLI, A. L; MARTIN, T. N.; DOURADO NETO, D.; REIS, A. R. Doses de Nitrogênio e Fontes de $\mathrm{Cu}$ e Mn suplementar sobre a severidade da Ferrugem e Atributos morfológicos do milho. Revista Brasileira de Milho e Sorgo, Piracicaba, v. 5, n. 2, p.192-201, 2006. 